\title{
IoT meets BPM: a bidirectional communication architecture for loT-aware process execution
}

\author{
Stefan Schönig ${ }^{1} \cdot$ Lars Ackermann $^{2} \cdot$ Stefan Jablonski ${ }^{2} \cdot$ Andreas Ermer $^{3}$
}

Received: 29 October 2018 / Revised: 31 October 2019 / Accepted: 21 February 2020 / Published online: 19 March 2020

(c) The Author(s) 2020

\begin{abstract}
Business processes are frequently executed within application systems that involve humans, computer systems as well as objects of the Internet of Things (IoT). Nevertheless, the usage of IoT technology for system supported process execution is still constrained by the absence of a common system architecture that manages the communication between both worlds. In this paper, we introduce an integrated approach for IoT-aware business process execution that exploits IoT for BPM by providing IoT data in a process-compatible way, providing an IoT data provenance framework, considering IoT data for interaction in a pre-defined process model, and providing wearable user interfaces with context-specific IoT data provision. The approach has been implemented on top of contemporary BPM modeling concepts and system technology. The introduced technique has evaluated extensively in different use cases in industry.
\end{abstract}

Keywords Process Execution · Internet of Things · Wearables

\section{Introduction}

Business process management (BPM) is considered as powerful technology to operate, control, design, document, and improve cooperative processes [1]. Processes are executed within application systems that are part of the real world involving humans, cooperative computer systems as well as physical objects [2]. Internet of Things (IoT) as well as cyber-physical systems (CPS), denoting the internetworking of all kinds of physical devices, have become very popular these days [3-6]. Data sets grow rapidly, in part because they are increasingly gathered by cheap and

Stefan Schönig

stefan.schoenig@ur.de

Lars Ackermann

lars.ackermann@uni-bayreuth.de

Stefan Jablonski

stefan.jablonski@uni-bayreuth.de

Andreas Ermer

aermer@maxsyma.de

1 Institute of Management Information Systems, University of Regensburg, Regensburg, Germany

2 Institute for Computer Science, University of Bayreuth, Bayreuth, Germany

3 Maxsyma GmbH \& Co. KG, Floß, Germany numerous information-sensing IoT devices such as mobile devices, aerial (remote sensing), software logs, cameras, microphones, radio-frequency identification (RFID) readers, and wireless sensor networks. Therefore, IoT contributes to the recent trend known as big data. Handling big data requires techniques with new forms of integration to reveal insights from datasets that are diverse, complex, and of a massive scale [7-9].

Process execution, monitoring and analytics based on IoT big data can enable a more comprehensive view on processes. Embedding intelligence by way of real-time data gathering from devices and sensors and consuming them through BPM technology helps businesses to achieve cost savings and efficiency.

Let us consider a production process where raw material is processed by different machines under the supervision of human operators. In case of product quality issues, manual human interventions are necessary. Additionally, operators must be aware of current sensor data to decide on tasks to be executed next. Such a scenario might be better manageable when closely linking digital production and machine data, such as regularly available in typical Industry 4.0 [10,11] scenarios, with human operators as enabled by the integration of IoT and BPM. Here, human activities can be triggered by a BPM engine through intermediate reactions to appropriate IoT real-time data in the underlying process model. Human 
operators can be notified seamlessly without loss of time on wearable devices while leveraging current context-specific information. As a consequence, the integration of IoT and BPM technology could lead to efficiency gains by reducing reaction time and enhance the quality of task execution.

In the literature, several concepts are emerging on combining IoT and BPM, e.g., monitoring running processes to align them with the state of physical devices and objects [12-15]. Still, there are many open challenges to be tackled [16-18]. In particular, the exploitation of IoT technology for system supported process execution is constrained by the absence of a common architecture that manages and standardizes the communication between both worlds.

In this paper, we present an approach that combines the recording and analysis of IoT and sensor data with the technology of process management. Here, our aim is to build the developed integration on top of existing BPM concepts and technical solutions. In summary, we propose an integrated approach for IoT-aware business process execution that exploits IoT for BPM by (i) providing IoT data in a process-compatible way, (ii) providing an IoT data provenance framework, (iii) considering IoT data for interaction in a pre-defined process model, and (iv) providing wearable user interfaces with context-specific IoT data provision. The approach has been implemented and evaluated extensively in different industry scenarios. The results show that the application of IoT-enhanced BPM leads to less machine stops because operators need less time to recognize work to be done. Furthermore, the use cases successfully highlight the bidirectional communication capabilities of the presented architecture.

This article is an extension of conference publication [19]. It extends the original article in various ways:

(i) the concepts have been extended in different directions, a.o. several possibilities to bridge the abstraction gap between IoT and BPM and, in particular, several possibilities to actively interact with IoT objects based on standard BPM technology.

(ii) the implemented software is presented in much more detail.

(iii) the related work section has been extended and discussed more thoroughly.

(iv) the evaluation section has been extended significantly toward the presentation and discussion of several application use cases.

This paper is structured as follows: Sect. 2 describes research background and fundamentals. Section 3 gives an overview of related work. Section 4 introduces the approach for a IoT-aware process execution that takes into account both passive information provision as well as active interaction possibilities. In Sect. 5, we describe the implementation of our approach based on well-known communication protocols. In Sect. 6, we evaluate our approach with several extensive application use cases in different fields of industry and Sect. 7 finally concludes the paper.

\section{Research background and fundamentals}

The Internet of Things (IoT) is the inter-networking of physical objects like (i) electronic hardware, e.g., sensors and actuators or (ii) humans using wearable digital devices like glasses and smartwatches [3,6,20]. Such connected things collect and exchange data between each other. IoT allows things to be controlled remotely across existing network infrastructures, including the Internet [6,21]. A business process is a collection of related events, activities, and decisions that involve a number of (human) resources [1]. To support processes at an operational level, a business process management system (BPMS) can be used. During process execution, a variety of information is required to make meaningful decisions. With the emergence of IoT, data are generated from physical devices sensing their (business/manufacturing) environment that reflects certain aspects of operative processes. A BPMS deals, a.o., with the enactment of process models that define the interplay between environmental circumstances (depicted as data values), characteristics of participants (depicted as resource assignments) and corresponding activities to be executed. We consider processes as explicit process representations (pre-defined models), which later are enacted.

\subsection{Research problem}

Recently, several researchers raised specific research challenges that need to be tackled in order to align IoT and BPM technology [16]. In this section, we explain a specific subset of those challenges that is tackled by the integration of IoT objects and a BPMS as it is described in the work at hand.

First of all, IoT sensors need to be placed in a processaware way in order to be able to collect and record all process relevant IoT data. Therefore, sensors need to be carefully placed at machinery and humans and be digitally accessible. The acquired process relevant IoT information needs to be up to date and current. It needs to be clear where the data stem from and where it has been used (cf. data provenance), as well as the quality of the data at hand needs to be ensured. It becomes necessary to find a way to annotate the IoT data origin and to use this (meta-)information in business process models.

Second, in many processes some activities require the interplay between human operators and software/hardware modules. There is an increasing use of mobile devices fostering the delivery of work items to the right users. Here, 

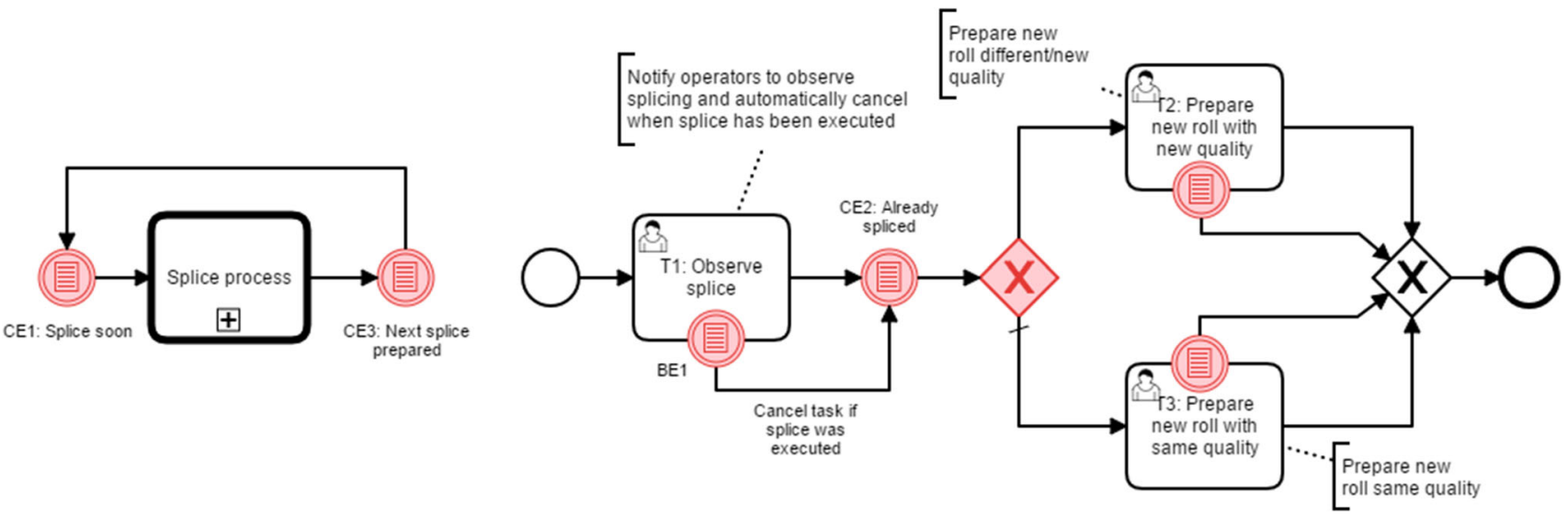

Fig. 1 Exemplary production process: a repeating subprocess; $\mathbf{b}$ splice process

an appropriate mapping from activities to user interfaces is needed allowing process participants to perform their work from arbitrary places in the working environment.

Third, participants might suffer from issues which hinder optimal working conditions. Here, the IoT can support the execution of tasks in a process through context-specific knowledge provisioning that is relevant for the users particular context. Sensor data can be leveraged to determine the actual context and to identify information needs.

These three challenges constitute the basis for the research of the work at hand. Accordingly, the underlying research question is how to design a BPMS within an IoT environment such that IoT devices like sensors and mobile devices are enabled to foster process execution in manifold terms like quality of task execution or reduction of delays. We tackle these open research gaps by conceptually designing and implementing a common technical architecture for IoTaware business process support. Sensing and perception the process environment via sensors constitutes the fundamental task of the IoT. Sensor data then must be aggregated, interpreted, and made available to the BPMS in order to trigger business process activities or human tasks, respectively. These tasks and corresponding context information must then be send in real time to responsible individuals that receive tasks on mobile user interfaces.

\subsection{Motivating example}

Additionally, we want to motivate the necessity of our approach by example of a real-life process from production industry that is visualized in Fig. 1. The example stems from the corrugation area where paper is glued to corrugated paper that depicts the basis for cardboard boxes. The example process is explained in detail in Sect. 6.1. The given example is a subprocess that is executed every time paper source rolls run empty, i.e., where new paper rolls need to be spliced with the paper from the low running roll. In order to effectively execute this process, several real-time interactions with IoT devices, i.e., sensors and operator equipment, are necessary: The BPMS must be aware of sensor data which indicates that a splice will happen soon, triggering the splice subprocess. Operators located somewhere along the up to 300 meters long machinery need to observe the splice process to avoid issues. Therefore, they need to be notified in real time to walk to the splicer. This requires wearable interfaces communicating with the BPMS over the IoT. Depending on a sensor value indicating the next roll quality, the BPMS has to execute different paths. In case the environment changes, operators tasks need to be reordered based on priorities or canceled by the BPMS. In addition to current tasks to be executed, operators require context-specific information at hand, e.g., the location of the splicer and the quality of the next paper roll. Furthermore, operators continuously need to observe viscosity and temperature of the glue to ensure a successful splice process. In the following sections, we show that the integration of IoT devices and a BPMS serves as a generalizable solution to the problems above.

\subsection{Research method}

This work is based on the Design Science Research (DSR) approach [22], which was adapted to the needs of this work. This approach is meant to solve identified problems in a build-and-evaluate process in order to create fitting information technology artifacts [23]. The hereby created artifacts are meant to be of relevance to a human purpose. The goal of creating and evaluating artifacts is to derive generalizable and transferable knowledge.

Typically, the DSR approach follows six iterative steps [24]: Initially, the problem needs to be identified and its importance needs to be shown (1). Second, objects of a solution are defined (2), and based on these objectives, the artifact is designed and developed in the third step (3). In the fourth step, the artifacts functionality is demonstrated (4) before 
Table 1 Overview on related approaches of the integration of IoT and BPM

\begin{tabular}{llllll}
\hline Approach & Mod. & Exec. & Mon. & UI & Context \\
\hline IAPMM [25,26] & $\checkmark$ & $x$ & $x$ & $x$ & $x$ \\
BPMN4CPS [27] & $\checkmark$ & $x$ & $x$ & $x$ & $x$ \\
BPMN for IoT [28-30] & $\checkmark$ & $x$ & $x$ & $x$ & $x$ \\
IoT/WS-BPEL [31] & $\sim(B P E L)$ & $\checkmark$ & $\sim(B P E L)$ & $x$ & $\sim(B P E L)$ \\
IoT/WS-BPEL [32,34,35] & $\sim(B P E L)$ & $\checkmark$ & $\sim(B P E L)$ & $x$ & $\sim(B P E L)$ \\
ADiWa[36] & $\sim($ conc. $)$ & $\sim($ conc. $)$ & $x$ & $x$ & $x$ \\
Extended GSM [2,12,13] & $\checkmark$ & $x$ & $\checkmark($ via GSM $)$ & $x$ & $\checkmark$ \\
This work & $\checkmark$ & $\checkmark$ & $\sim(B P M S)$ & $\checkmark$ & $\checkmark$ \\
\hline
\end{tabular}

it gets evaluated (5), determined by the evaluation criteria and objectives of step two. Therefore, different evaluation approaches depending on the specific use case are possible [23]. Finally, the results are communicated and published (6).

We strictly followed the above-described iterative steps in the work at hand. In the first step, we identified significant needs for combining IoT and BPM technology. This has been shown by concrete challenges defined by the current scientific literature (cf. Sect. 2.1) as well as by means of a concrete example scenario (cf. Sect. 2.2) (1). In the second step, we derive specific design objectives that our IoT-aware process architecture needs to fulfill (2). These objectives determine the functionality and design of the prototype (3) that are presented in Sect. 4 and implemented in Sect. 5 of the work at hand. We demonstrated the functionality of our prototype in several, extensive real-life use cases (4). We conducted an evaluation in terms of usability studies and performance improvements (5). Finally, we conducted step six by presenting our results on several conferences and submitting this manuscript.

We also met the requirements of DSR as an iterative process [24] where we not only conducted rapid prototyping of the system, but also presented intermediate results to the industry partners. Their input and knowledge were used to additionally refine our objectives and therefore the design and functionality of the system architecture.

\section{Related work}

Several approaches have been proposed to relate IoT objects with business processes. An overview of related approaches for IoT and BPM integration is given in Table 1. The table summarizes the support of each approach for IoT-aware process modeling, execution, and monitoring as well as the availability of a mobile user interface and the possibility to provide (IoT) context information. In [25,26], the authors present the Internet-of-Things-Aware Process Modeling Method (IAPMM) that covers requirements analysis.
It extends the BPMN meta-model to model IoT-aware processes. The approach in [27] (BPMN4CPS) also describes an extension of BPMN in which the process logic is split into the cyber part, the controller, and the physical part. Furthermore, the authors extended BPMN by new task types. Some more notation concepts in BPMN for IoT are described in [28-30]. The main focus is on the modeling of real-world properties. Also in [28,29] the authors present an extension of BPMN with new modeling concepts. None of the described approaches provides details on how to execute these models. In [31], an approach for implementing an IoT-aware execution system given in WS-Business Process Execution Language (WS-BPEL) is introduced. It extends BPEL by context variables which are automatically updated. The authors implemented a prototype which is compliant with every WS-BPEL engine. Other approaches implementing BPEL extensions are presented in $[32,33]$. The variables are updated using the publish/subscribe paradigm. Another extension for WS-BPEL (Context4BPEL) with features to manage context events to allow the asynchronous reception of events, query context data and evaluate transition conditions based on context data is described in [34]. In [35], the authors integrate distributed resources into WS-BPEL by formalizing a fragment of WS-BPEL together with the WSRF (Web Services Resource Framework). In [36], the authors propose an approach for enabling IoT-based agile business processes. They provided concepts for extending models by triggers for variance. The approaches in $[2,12,13]$ rely on the information coming from artifacts involved in the process to understand how a process evolves. By adopting an extension of Guard-Stage-Milestone (GSM), it is possible to monitor the process even when the control flow is not respected. The work presented in [37] introduces a lightweight process engine for enabling mobile applications. The authors describe requirements and concepts for mobile process applications and a prototypical mobile user interface. However, this work does not comprise IoT related aspects.

The work presented in [17] lists and reviews specific challenges and opportunities to combine BPM and complex event processing (CEP) technology. These challenges are consid- 
ered as the core issues of this combination. The authors suggest that addressing these issues would form a basis for future applications. Therefore, this work can be seen as a motivation for the article at hand.

In addition to the stream of research that integrates IoT and BPM concepts, the work at hand is related to research on (mobile) task assistance and decision support systems in production area, i.e., concepts and approaches related to the term Industry 4.0 [10,38]. Here, sensors have the capability of measuring a multitude of parameters frequently and collecting plenty of data. Analysis of big data, both historical and real time, can facilitate predictions on the basis of which proactive maintenance decision making can be performed $[39,40]$. The e-maintenance concept can significantly address these challenges. There are several research works that introduce intelligent agents that are directly implemented at the shop floor level [41]. Furthermore, there is research about web-based production maintenance services and architectures that include wireless sensing of process data and identification technologies, data and services integration and interoperability [42]. Portable computing devices have been used for production monitoring for many years. Though initially offered as an integrated instrumentation solution, mobile devices such as PDAs and tablets have been programmed with a mobile capacity to analyze and present data, disconnected from the actual sensing components $[43,44]$. These solutions introduce concepts, architectures and prototypical implementations for configuring the sensing infrastructure and for presenting certain process and equipment data on mobile devices. The work at hand, however, extends the described solutions by introducing an integrated sensor and process-based information system that is implemented on top of wearable operator interfaces.

\section{Bidirectional architecture for an loT-aware BPMS}

Based on the three challenges defined in Sect. 2.1, we can define requirements of the corresponding artifact that will be developed throughout this work.

We propose a four-steps procedure to provide the necessary information. The first step is connecting IoT objects and their values traceable to a BPMS. We call a single type of value of a certain (sensor) object variable. The second step is extending a BPMN process model with data variables participating in the process stemming from physical objects such as machine status or actor positions. The resulting process models must be applicable by default contemporary BPMN execution engines. This way, organizations can reuse existing process models, without having to learn new languages and remodel processes from scratch. The third step is to establish a real-time notification interface of triggered activities to pro- cess participants by means of mobile devices. In the fourth step, context relevant information stemming from connected objects must be selected and provisioned to users.

Summing up, our approach focuses on the following open research gaps defined in the literature [16]:

- IoT sensors need to be placed in a process-aware way and be linked to running processes $\left(C_{1}\right)$.

- Mobile devices should be used, fostering the delivery of work items to the right users $\left(C_{2}\right)$.

- IoT should support the execution of tasks through context-specific knowledge provisioning $\left(C_{3}\right)$.

Based on these challenges, our approach poses the following four main requirements that can directly be linked to the challenges mentioned above:

R1. A BPMS must be aware of current values of IoT objects. (cf. $C_{1}$ ) Variable attributes, e.g., address and identifiers, must be configurable and traceable, i.e., it must be clear where the data stems from. The acquisition of current values from diverse data variables with standard IoT protocols must be possible. Here, erroneous data must be detected and excluded. Based on an established mapping from IoT variables to process models, IoT data must be sent to a BPMS.

R2. Each defined variable must be referenceable in the executed process model. (cf. $C_{1}$ ) Based on current values of certain variable, tasks are triggered or canceled and decisions are made.

R3. Responsible users must be notified on mobile devices in real time. (cf. $C_{2}$ ) Process participants must be seamlessly notified when human interaction is required, independent of where the user is located.

R4. Context-specific knowledge must be provisioned to users. (cf. $C_{3}$ ) Alongside with activity notification, context-specific and process relevant information must be provisioned to users.

\subsection{Connecting current data of loT objects to BPMS}

In this section, we describe the necessary steps to connect the IoT objects and their current values to BPMS. Here, we focus on a variable mapping and discuss different methods to bridge the existing abstraction gap between IoT data and business processes.

\subsubsection{Variable mapping}

First, we formally define IoT object data that are regularly, i.e., in a certain interval int acquired from digitally accessible devices and stored in a IoT middleware 
Fig. 2 Acquisition: mapping and connecting current IoT variables to BPMS

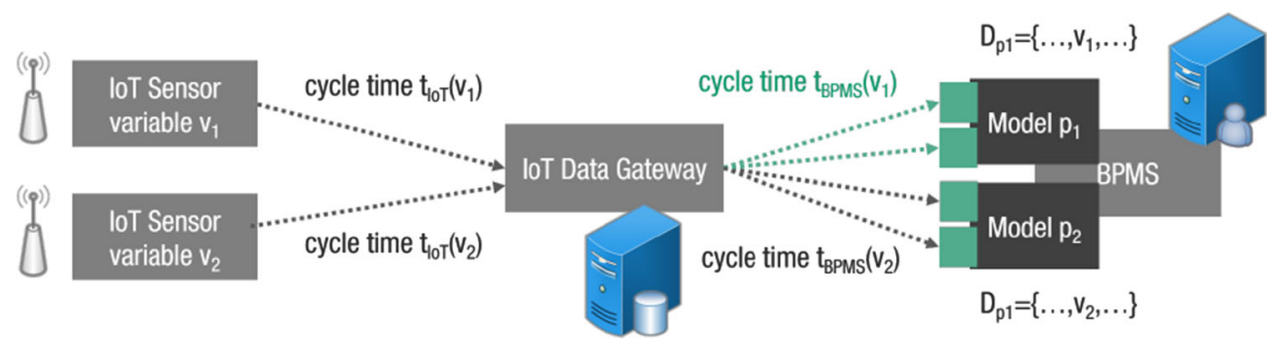

database. Data recorded from IoT objects are an ordered sequence $\sigma$ of events $e_{j}$, i.e., $\sigma=\left\langle e_{1}, \ldots, e_{|\sigma|}\right\rangle$. In general, an event $e$ is defined as tupel of attributes-value pairs, i.e., $e=\left(\left(a t t r_{1}^{e}, v a l_{1}^{e}\right), \ldots,\left(a t t r_{|e|}^{e}, v a l_{|e|}^{e}\right)\right)$. The set of all attributes $A T T R^{e}$ and the corresponding values $V A L^{e}$ of an event $e$ are therefore defined as $A T T R^{e}=$ $\left.\left\{\operatorname{attr}_{1}^{e}, \ldots, a_{t t r}^{e}\right\}\right\}, V A L^{e}=\left\{v a l_{1}^{e}, \ldots, v_{|e|}^{e}\right\}$. Each $a t t r_{i}^{e}$ has an unique variable identifier $v$ and a timestamp time, i.e., $\forall_{e \in \sigma}\left(v^{e}, t i m e^{e}\right) \subseteq A T T R^{e}$. Each attributes-value pair $\left(a t t r_{i}^{e}, v a l_{i}^{e}\right)$ is dedicated to exactly one IoT variable. With $l^{e}\left(v_{i}^{e}\right)=v_{a l}^{e}$ we denote the value $v a l_{i}^{e}$ of a certain variable $v_{i}^{e}$ in event $e$. A total order of events is implemented as follows: $\forall_{1 \leq j<k \leq|\sigma|}\left(t i m e^{e_{j}}\right)<\left(t i m e^{e_{k}}\right)$. Therefore, the current value of a certain variable $v_{i}$ is given as $l^{e}\left(v_{i}^{e|\sigma|}\right)=$ $v_{a l}{ }_{i}^{|\sigma|}$. All other attributes are optional. Based on these definitions, we ensure that IoT data variables are configurable and traceable (cf. R1). In the next step, current data of each connected IoT object must be sent to the BPMS (cf. R1). Therefore, we need to map IoT data to enacted process models of the BPMS. Consider a set of process models $P$ where each $p$ contains a set of data variables $D_{p}$. Each variable $d \in D_{p}$ has an unique identifier $v_{d}$. The underlying assumption is that each participating IoT variable is referenced by the same unique identifier in the corresponding process model. If we want to establish a connection between the data of an IoT object and a process variable, then both identifiers have to be the same, i.e., $v_{i}=v_{d}^{p}$. As visualized in Fig. 2, IoT variable $v_{1}$ is semantically mapped to variable $v_{1}$ of process model $p_{1}$ and IoT variable $v_{2}$ to variable $v_{2}$ of process model $p_{2}$, respectively.

\subsubsection{Bridging the abstraction gap}

Having established such a semantic mapping, only current data of each connected object are sent to the BPMS, i.e., a sending procedure $s p$ is initiated for each variable $v_{i}^{e_{|\sigma|}}$ recorded in $e_{|\sigma|}$ sending the latest acquired values to the BPMS, i.e., $\forall_{1 \leq i \leq|e|} v_{i}^{e_{|\sigma|}}$. The technical implementation of the connection is hereby established by means of a IoT infrastructure. Here, it is frequently necessary to bridge a certain abstraction gap between partly high frequent IoT and sensor data and human activities of business processes. We outline three different possibilities to bridge the gap. Note that it is
Table 2 Example acquired IoT object data

\begin{tabular}{llll}
\hline Event & attr $_{i}^{e}(v, \ldots$, time $)$ & val $_{i}^{e}$ & Data type \\
\hline 1 & $\left(R M_{1}, \ldots, 2018-01-0910: 15: 32\right)$ & 300 & Historic \\
1 & $(Q U, \ldots, 2018-01-0910: 15: 32)$ & $211 \mathrm{C}$ & Historic \\
1 & $(G T, \ldots, 2018-01-0910: 15: 32)$ & 120 & Historic \\
2 & $\left(R M_{1}, \ldots, 2018-01-0910: 15: 42\right)$ & 133 & Current \\
2 & $(Q U, \ldots, 2018-01-0910: 15: 42)$ & $211 \mathrm{C}$ & Current \\
2 & $(G T, \ldots, 2018-01-0910: 15: 42)$ & 115 & Current \\
\hline
\end{tabular}

only necessary to clearly mention the concepts, and all of them are ready for use in contemporary IoT platforms that are based on streaming environments like Apache Spark., ${ }^{1}$ Apache $\mathrm{Kafka}^{2}$, etc.

Simple cycle time to cycle time method The simplest method is probably a simple cycle time to cycle time mapping between the IoT world and the BPM world. Figure 2 visualizes this mapping scheme by providing different cycle times. IoT data are gathered and stored with high frequency (cycletime $_{I o T}$ ) while the same value is delivered to a BPMS with lower frequency (cycletime $B$ PMS ).

Example: Let us assume that we acquire data from a sensor indicating the restmeters of paper on a specific roll $R M_{1}$, the quality of the paper roll to be used next $Q U$ and the current glue temperature $G T$ from a temperature sensor in an interval $i n t=10 \mathrm{sec}$. The acquired IoT data are then exemplarily given as shown in Table 2 . Consider a process model $p$ where two data variables are referenced and evaluated. These variables are defined as $v_{d_{1}}^{p}=R M_{1}$ and $v_{d_{2}}^{p}=Q U$, respectively, in the model. The procedure $s p$ is initiated every 10 s. Therefore, the last execution of $s p$ sends the values 133 and $211 C$ to the BPMS where these values are dedicated to the corresponding process variables $\left\{d_{1}, d_{2}\right\} \in D_{p}$. Note that data referring to $G T$ are not sent to the BPMS.

Condition and aggregation-based method A more sophisticated method to bridge the abstraction gap between both worlds is to introduce conditions, triggers and aggregations.

\footnotetext{
${ }^{1}$ https://spark.apache.org/.

2 https://kafka.apache.org/.
} 
Here, IoT data are only sent to a BPMS if certain conditions hold true. Note that these conditions must not be related to the IoT data only, and they can also refer to specific data value from other sources. Examples are, a.o., (i) IoT data are sent at a specific point of time, (ii) IoT data are sent if the current value is below a certain threshold, or (iii) IoT data are sent if a certain external variable is below a certain threshold. Considering that a certain time elapsed between recording IoT data and the point of time where it was sent to the BPMS, it might be necessary to first calculate aggregations on the recorded data before sending the result to the BPMS.

Complex event processing-based method The most elaborate approach to bridge this gap is to make use of complex event processing methods [17]. Here, the simple conditionbased method is replaced by a more complex technique where we look at recorded IoT data in more detail. A single data acquisition record is seen as an event where each event has specific attributes, e.g., value or timestamp. In CEP, we can define patterns on these attributes that have to hold in order to trigger a sending procedure. Here, a potential pattern might be: sent IoT data to the BPMS if the considered sensor sends $n$ values below threshold $x$ in a time window $t$.

\subsection{Enrichment of process models with loT variables}

As described in Sect. 4.1, we consider a set of process models $P$ where each $p$ contains a set of process variables $D_{p}$ that semantically correspond to a variable of an IoT object. Based on the established real-time connection described before, these IoT-aware process variables, identified by $v_{d_{i}}^{p}$, can be referenced in enacted process models in various ways. We assume that a given process model $p$ is defined as BPMN conform process diagram, one of the most used formalisms for process modeling, representing the IoT-aware process to be executed. A BPMN process diagram specifies which activities are executed in a process as well as their control flow relationships. To be able to infer when activities start or end based on the state of the variables, the diagram must capture this information (cf. R2). This step requires the process designer to enrich the given BPMN diagram by including information on how and where the connected IoT variables influence the process. Here, data of IoT variables can only be read, i.e., passive, informing or be actively written, i.e., active, interacting.

\subsubsection{Passive informing}

When using IoT object data in business process models, we consider several possible interaction possibilities. The possibilities described below can serve as a good starting point to understand the concepts and potentials of enacting IoT information in process models. a. IoT-based trigger events Sometimes, we only want a process to start or to continue if a certain condition is true. In BPMN conditional events, define an event which is triggered if a given condition is evaluated to true. It can be used as an (i) Intermediate Conditional Event, as a (ii) Boundary Event or as a (iii) Conditional Start Event. In case of (i), current values of used IoT variables $\operatorname{var}_{d_{i}}^{p}$ trigger an Intermediate Catch Event and the execution continues to the next activity. In case of (ii), the BPMS checks whether the process environments changed based on the current values of the connected IoT variables. If the given condition is satisfied, then the corresponding activity will be interrupted.

Example: Consider the example process in Fig. 1 where the control flow reaches the conditional event $C E 1$ and the condition is, for example, $R M_{1}<200$. Given a current IoT sensor value of $v^{2} l_{R M_{1}}^{e}=133$, the condition will be satisfied and the BPMS triggers the subprocess Splice process. Similarly, if the splice has been executed successfully, i.e., $\mathrm{val}_{R M_{1}}^{e_{|\sigma|}}>200$, then Observe splice is aborted by means of the corresponding boundary event $B E 1$.

b. IoT-based decisions In BPMN, an data-based gateway is used to model a decision in the process. Similar to conditional events, current IoT variable values can be used to decide which sequence flow is selected for continuing the process.

Example: Depending on the next paper quality to be used in the production order, the BPMS has to decide how to continue the process, i.e., based on the current value of $v a l_{Q U}^{e_{|\sigma|}}$ either task $T 2$ or task $T 3$ will be triggered.

c. IoT-based loops In order to model repeated behavioral patterns, IoT variables can be used to define event-driven loops. This way, end-to-end processes can be broken up into comprehensible micro-processes.

Example: In order to support the recurring splice process in Fig. 1a, the subprocess is surrounded by the conditional events $C E 1$ and $C E 3$. While the positive evaluation of the given conditions in $C E 1$, i.e., splice happening soon, triggers the subprocess to be executed, $C E 3$ checks if all preparations for the next splice have been executed. In case the corresponding IoT stemming data values fulfill the given event condition, the process continues $C E 1$.

\subsubsection{Active interaction}

In order to establish a full-fledged integration between IoT objects and process management, it is necessary to also provide an active interaction possibility, i.e., write access from a BPMS to IoT objects. As for the passive informing case, the BPMN language already provides several language elements that can be used to establish such a communication. Service Tasks are used to call (web) services or program code in general and are therefore a solution for defining automatable tasks. Contemporary BPMS is often already equipped with fully implemented connectors to established web communi- 


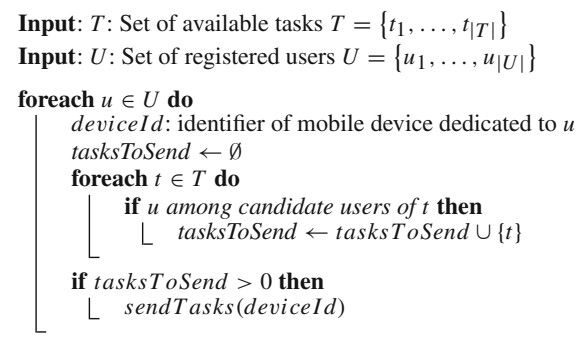

Algorithm 1: Distributing assigned human tasks to wearable devices

cation protocols like HTTP. On the other hand, IoT objects frequently provide open interfaces for protocols like HTTP such that a direct communication interface from BPMS to IoT object can be realized. However, also in application scenarios where IoT objects are only accessible by means of different extrinsic protocols (a.o. TCP/IP, MQTT, etc.), it is possible to establish an integration. Here, we make use of diverse open-source programming tools for wiring together hardware devices like the Node-RED platform. ${ }^{3}$ This way, e.g., a BPMS communicates via HTTP with a wiring platform that, in turn, handles the request and creates a message according to the target protocol of the IoT object.

\subsection{Establishing the real-time mobile user interface}

It is important that process participants are seamlessly notified when human interaction is required, independent of where the user is located. This requires a real-time notification on mobile devices of responsible users (cf. R3). Therefore, it is necessary to define a mapping of users to corresponding mobile devices that serve as wearable, userspecific process cockpits and task lists, respectively. This is achieved by specifying a dedicated mobile device identifier for each defined user in the BPMS. During process execution, the currently available tasks for a specific process participant are then directly sent to the specified mobile device. The algorithm to distribute the currently available human tasks to defined mobile devices is given in Algorithm 1. The actual operator to device mapping and the task distribution is described in detail in Sect. 5.

Example: The splice process in Fig. 1b requires operators to manually observe the splice at the specific machinery $(T 1)$. Therefore, in case the condition in $C E 1$ evaluates true, the responsive operator needs to be notified in real time to be able to walk to the splice site in time. Hence, as soon as task $T 1$ becomes available, the list of currently available tasks of assigned operators implemented as a smartwatch application is updated.

\footnotetext{
${ }^{3}$ https://nodered.org/.
}

\subsection{Context-specific information provision}

Alongside with activities, context-specific and process information must be provisioned to operators to improve the quality of task execution (cf. R4). In order to ensure that the provisioned information is of value for operators, the following three dimensions need to be considered when defining data that should be delivered to certain process participants. Based on these dimensions, Algorithm 2 distributes IoT information in a context aware way to corresponding users.

a. Dedicated Context-Which entities allow for a separate context definition? There are different entities where different contexts can be defined for. IoT variable information can be dedicated to subprocesses and delivered alongside with tasks of this subprocess to respective users. Furthermore, IoT variable data can be dedicated to specific user groups or roles. Within a location-aware BPM scenario, the information context can also be defined based on locations.

b. Information/Source-Which IoT information should be provisioned? IoT data can be classified according to the factors of the context framework defined in [45], i.e., intrinsic or extrinsic context information. Intrinsic IoT variable information reflects data used in the process model and is therefore directly related to the currently executed process instance. Extrinsic IoT data are information that is not necessarily related to a running processes but might influence process execution as well, e.g., production hall temperature. Furthermore, the granularity of provisioned information must be adjustable w.r.t. different processes. In some cases, it might be helpful to see more information; in some cases, it might be better to see less information to prevent users from information overload.

c. Visualization-How is a certain context-specific IoT variable presented or visualized? Context-specific IoT information must be visualized in a proper way. Depending on the class of information (intrinsic, extrinsic) or the data type (string, number) different positions on the interface and representation styles are appropriate. Intrinsic, instance-specific information that is relevant for the execution of a certain activity can be represented as information below the activity name. Extrinsic, environmental data that might be of importance to process execution can be represented as separate controls on additional tabs.

Example: In the splice process in Fig. 1b, a context is defined for specific groups of operators. Only the users assigned to group Wet-End will receive information w.r.t. the splice process. Members of this group receive intrinsic, instance-specific information like the quality of the paper roll to be used next. Since this is highly relevant data for executing task $T 2$, this information is presented directly below the activity name. Furthermore, users receive the restmeters of paper on a specific roll (intrinsic) and the current glue temperature (extrinsic). Both values are numbers that are important 


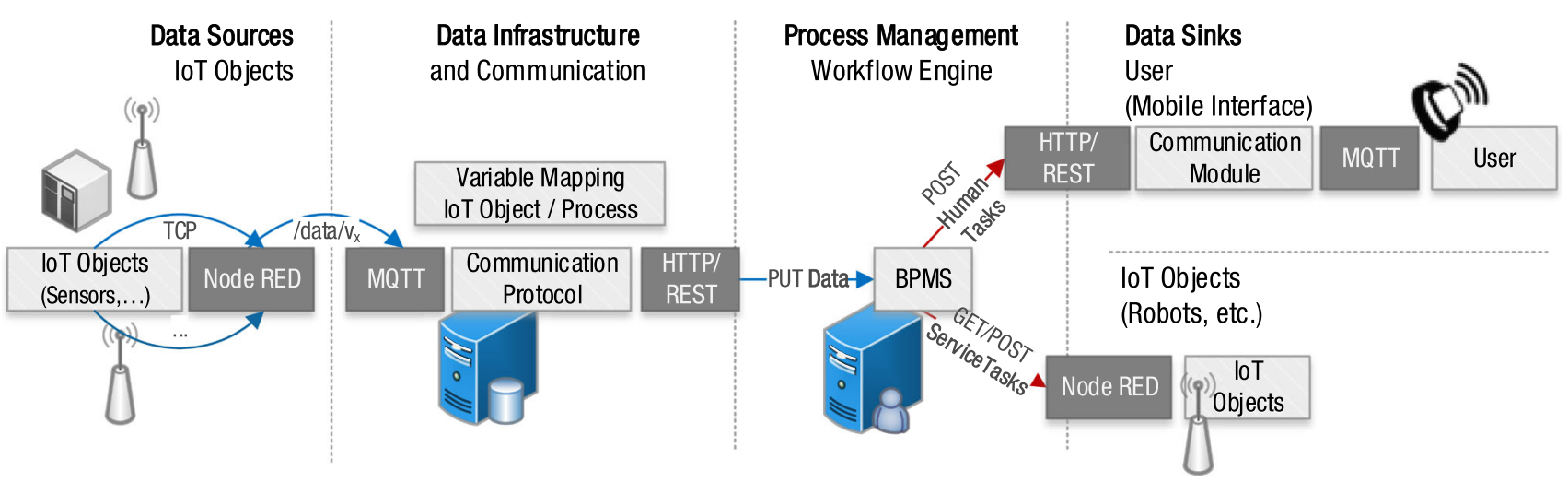

Fig. 3 Integrated communication architecture for IoT and BPMS

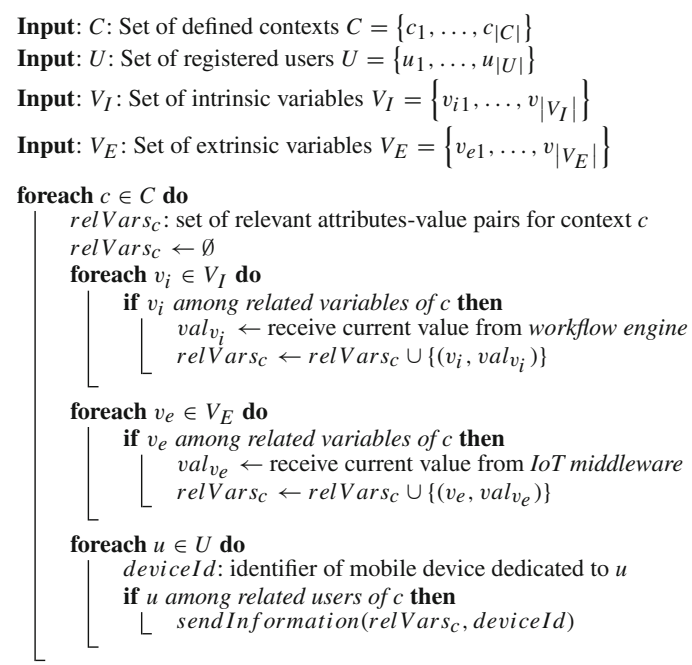

Algorithm 2: Provisioning context-specific IoT information

information for operators but not directly related to activities. Thus, these values are visualized on additional tabs on the operators smartwatch.

\section{Architecture and implementation}

The described approach has been implemented based on a four layer architecture that is visualized in Fig. 3. It consists of the following layers: (i) IoT objects like sensors as data sources; (ii) IoT infrastructure and communication middleware; (iii) the BPMS; and (iv) data sink in form of IoT objects of human process participants. The layers are connected based on standard communication protocols.

\subsection{IoT data acquisition and BPMS integration}

In order to connect arbitrary IoT objects, we make of the open-source platform Node-RED of IBM. The platform acts as a communication middleware between various IoT protocols and data sources like TCP sensors and the BPMS. To allow the IoT objects at layer (i) to communicate with the IoT middleware at layer (ii) and the BPMS, respectively, a Message Queue Telemetry Transport (MQTT) ${ }^{4}$ broker is used. MQTT is a queue-based publish/subscribe protocol, which is especially suited for applications where computing power and bandwidth are constrained. The used MQTT topics are listed in Table 3. IoT objects, i.e., sensors or actuators, represent publishers. They are connected to an IoT gateway using specific architectures such as Profibus, LAN, WLAN, or Bluetooth. A specific IoT variable $v_{x}$ is acquired and published on a MQTT topic $/ v_{x} /$ data . Through a MQTT broker, the acquired data are sent to an acquisition application at layer (ii) that stores IoT data into a high-performance NoSQL database that follows the database scheme described in Sect. 4.1. In our implementation, we used the latest version of the Apache Cassandra database.

A distribution application at layer (ii) keeps the BPMS updated with the latest IoT values. All running instances of a particular process receive the corresponding data value. The application cyclically acquires the values from the database in a key-value structure and sends them to the BPMS. The configuration interface of the distribution application is given in Fig. 4. On the left-hand side, the screenshot shows different connected IoT variables and their specific acquisition setting on the top of the window. The section at the bottom contains the configuration settings for the mapping to process variables and the relevant process models. In our architecture, we used the latest version of the Camunda BPMS and therefore communicated with the workflow engine by means of the Camunda Rest API, ${ }^{5}$ i.e., PUT, POST and GET HTTP requests as described in Fig. 3. The tools at layer (ii) ensure that process relevant information stemming from the IoT is up to date. Through the acquisition tool, IoT data meta infor-

\footnotetext{
${ }^{4} \mathrm{http} / / /$ docs.oasis-open.org/mqtt/mqtt/v3.1.1/mqtt-v3.1.1.html.

5 https://docs.camunda.org/manual/7.8/reference/rest/.
} 
Table 3 MQTT communication between IoT objects and BPMS

\begin{tabular}{lll}
\hline Topic & Description & Direction \\
\hline$/\{$ variable_id\}/data & IoT sensor data & IoT to BPMS \\
$/\{$ actor_id\} & Device configuration & BPMS to IoT \\
$/\{$ actor_id\}/tasks & Tasks of specific actor & BPMS to IoT \\
$/\{$ actor_id\}//variable_id\} & Context data for specific user & BPMS to IoT \\
$/\{$ actor_id\}/command & Actors actions (claim, complete, cancel) & IoT to BPMS \\
$/$ keepalive & & IoT to BPMS
\end{tabular}

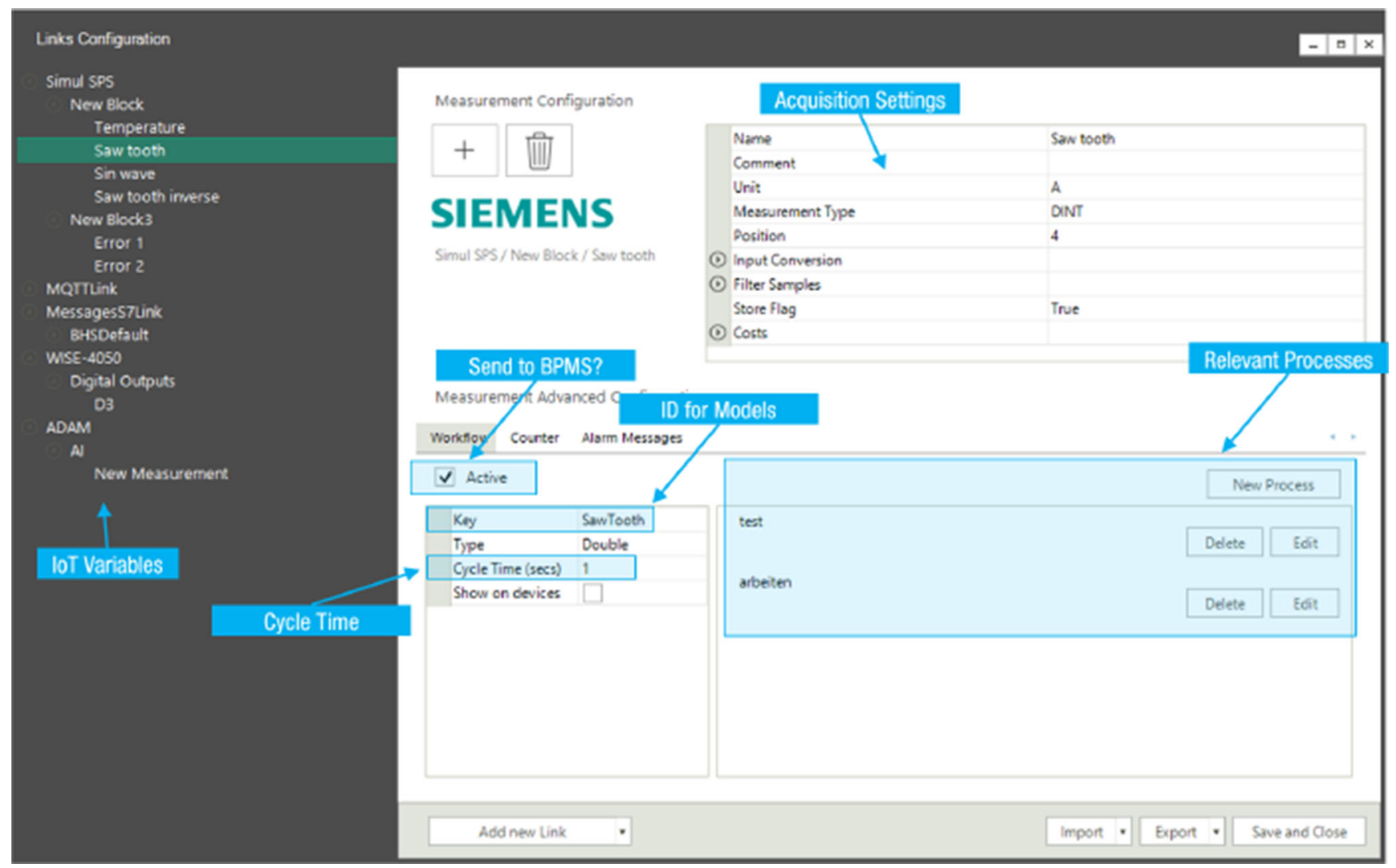

Fig. 4 Configuration interface from IoT data variable to process model variable mapping

mation is provided that makes clear where the data stems from. Given the current IoT data values, the engine calculates available activities.

\subsection{User interface and loT object interaction}

As a mobile user interface, we implemented an Androidbased smartwatch application that subscribes to specific MQTT topics. The distribution application cyclically requests the current user tasks from the Camunda API for each defined user and publishes to the correct MQTT topic, given the mobile device identifier, i.e., smartwatch device, configured on the BPMS (cf. Algorithm 1). The application allows users to start and complete tasks as well as to initiate new process instances. The process of the device recognizing its configu- ration is implemented as follows: the distribution application cyclically checks the user configuration in the BPMS. When a change is detected, it publishes the new configuration to the topic / $\{$ actor_id\}. The assignment of a smartwatch to a specific user is implemented by means of a unique device id, i.e., the smartwatch of a certain actor subscribes to the topic of its specific device identifier. Having established such connections, the smartwatch communicates with the MQTT broker by subscribing to the following topics: The current tasks for a specific operator are published on the topic/\{actor_id\}/tasks. The device sends operators commands, such as complete task to the topic/\{actor_id\}/command. The content of the message is forwarded straight to the BPMS using a POST request. Context-specific IoT data are sent to actors on topic $/\{$ actor_id\} $/\{$ variable_id\} based on Algorithm 2. To prevent 
the MQTT service at the watch to be killed, we implemented a keep alive communication (topic $=$ /keepalive).

In case of active interactions with the IoT environment, BPMN Service Tasks are used. Here, we make use of the Camunda HTTP connector either to directly communicate with IoT objects that support HTTP communication or to send current variable values to Node-RED. An example sheds light on the complex architecture: Let us assume that after the completion of a certain human task a robot must be called to a specific position. This position is transferred as a parameter in a HTTP request send from the BPMS to the Node-RED platform. In Node-RED, the parameter is read from the body of the request and transformed to a message format according to a protocol that the corresponding robot can process. This way, the presented solution implements a full-fledged bidirectional communication between IoT objects like sensors, smartwatches or robots and a BPMS.

\section{Evaluation and industrial applications}

In this section, we describe the extensive evaluation of the proposed approach by means of several different application scenarios in industry. First, we present the application carried out in production industry. Second, we describe the use case of integrating human tasks and automated robot activities. Finally, we describe the process-based implementation of the SEMI E10 specification for definition and measurement of equipment reliability, availability, and maintainability. ${ }^{6}$ Every use case can be characterized according to the following scheme: (i) type of acquired IoT data (cf. Sect. 4.1.1 and Sect. 4.2); (ii) type of IoT data abstraction (cf. Sect. 4.1.2); and (iii) type of IoT data sinks (cf. Sect. 4.2.2 and Sect. 4.3). Our evaluation in different industry use cases has been carried out to show that our architecture works and can be applied in various use cases. Each use case provides a different implementation and view on the architecture. According to the Design Science approach, we created generalizable and transferable knowledge that can be applied in various application areas.

\subsection{Application in production industry}

The first use case has been implemented within a production industry scenario. More precisely, we introduced BPM support for several production processes of production industry plants where paper is glued together to produce corrugated paper as raw material for cardboard boxes. Due to increasing automation and staff reduction, less operators are available to control a corrugated paper production line. Hence, interactions between users and machinery requires several

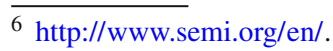

location changes of users between control panels that result in delayed information flows. These delayed reaction times are frequently the reason for increased deficient products. Furthermore, corrugation plants currently have to face a high fluctuation of employees such that process knowledge is lost over time. As a result, frequently new employees have to learn a basic understanding of production process control from scratch. Based on these issues, the general goals of the project carried out are (i) to increase operators productivity in terms of reducing stop times and increasing production speed, (ii) to facilitate the education and onboarding of new employees through transparent process knowledge and, (iii) to ensure traceability of work steps. The described solution has been rolled out in different plants. In total, forty production operators have been equipped with smartwatch devices and assigned a user in the BPM system. This first application case can be characterized as follows:

(i) Acquired IoT data: data from diverse PLCs (Siemens S7 communication protocol) and sensors according to the MQTT protocol

(ii) IoT data abstraction: cycle time mapping; acquisition of IoT data three times per second; distribution to BPMS every second

(iii) IoT data sinks: wearable smartwatch interface for operators' human tasks connected via MQTT protocol

The overall BPMN process that is executed by a Camunda BPMS can be described as follows: After initializing internal helper variables, the control flow splits into different parallel paths where each path calls a specific subprocess depending on certain IoT-based sensors conditions to be fulfilled. Each of these subprocesses reflects necessary operations that need to be performed to control production, e.g., the splice subprocess in Fig. 1. The implemented process contains all IoT-enhanced modeling concepts that have been introduced in Sect. 4.2. To directly notify operators when human actions are needed, plant personal has been equipped with smartwatches, i.e., mobile process cockpits as described before and as shown in Fig. 5. Therefore, a user-group model has been defined in the BPMS. Here, available operators were assigned to a specific area of production that depicts their area of responsibility. Thus, depending on the area operators are working, the BPMS assigns a different set of tasks. Operators are then pointed to new human tasks through visual, acoustic and, in case of noisy environments through haptic signals like vibration alarms. Furthermore, operators are used more effective because low priority work is aborted in order to perform high-priority work that could lead to machine stops.

In addition to currently available human tasks the IoT infrastructure provides diverse context-specific information on the smartwatch interface of operators. Depending on the 


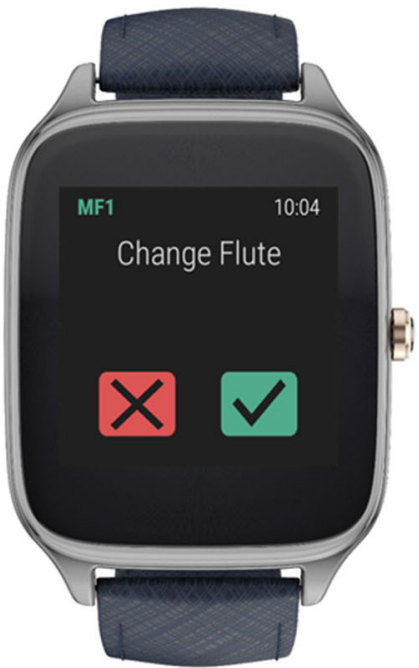

(a)

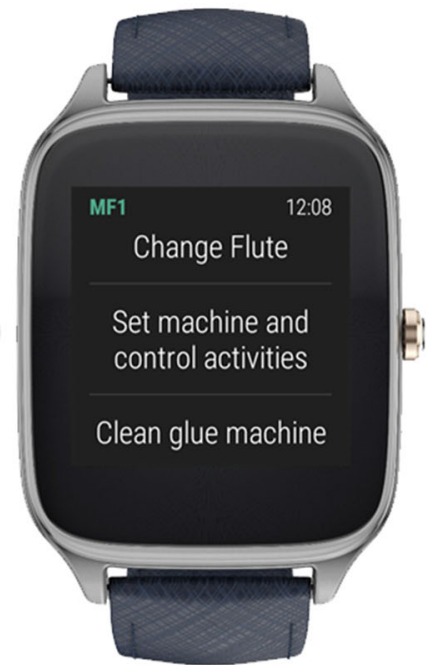

(b)

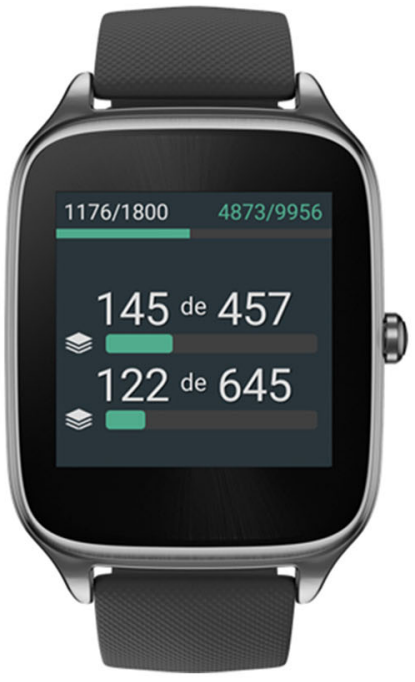

(c)

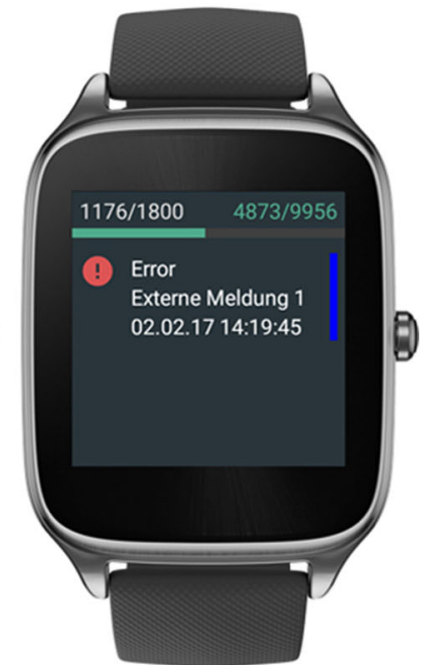

(d)

Fig. 5 Wearables: a unclaim/complete task; $\mathbf{b}$ tasks; $\mathbf{c}$ and $\mathbf{d}$ context info

specific group, a user is assigned to, the wearable device offers diverse context information to operators: at the DryEnd (the area where produced corrugated paper leaves the plant), e.g., the remaining time of current production job, the remaining time to next stack transport, or the current production speed. Users at the Wet-End (the area where original paper is inducted to the plant) receive continuously information w.r.t. the next necessary roll change or occurring error and defects of machinery modules. In addition, operators receive error messages and environmental information from the different plant modules. This way, concrete and goaloriented information in error cases or warning messages for supply shortfalls can be transmitted to operators and enhance the over all process transparency and thus the quality of task execution (cf. Challenges in Sect. 2.1). Through the described implementation, it was possible to significantly reduce reaction time intervals. The amount of deficient products was decreased and the overall quality of the produced corrugated paper has been improved. The overall equipment downtime was significantly decreased, since problems have been prohibited or recognized in advance and were solved proactively. Hence, the overall equipment efficiency could be increased effectively. To quantify these findings, we analyzed process execution with the Camunda Statistics Plugin. ${ }^{7}$ We tracked the corrugation process (i) for five days without operators using wearable devices and (ii) other five days with operators being notified using smartwatches. In particular, we measured the average instance throughput time for a splice processes. The effectiveness of the approach has been measured based on machine stop times and waste reduction. On average, 100 splices are executed per shift, i.e., $8 \mathrm{~h}$ of pro-

\footnotetext{
${ }^{7}$ https://github.com/camunda/camunda-cockpit-plugin-statistics.
}

duction. In case (i), we recorded a total stop time of $180 \mathrm{~min}$, i.e., on average $12 \mathrm{~min}$ per shift. In case (ii), the stop time has been decreased to $60 \mathrm{~min}$ in total, i.e., 4 min per shift on average. The results show that the application of the IoTenhanced BPMS leads to less machine stops because users need less time to recognize work to be done.

\subsection{Application for robot human interaction}

The second use case has been implemented with a robot that delivers certain drinks when the respective process is initiated by a human operator. The second application case can be characterized as follows:

(i) Acquired IoT data: robot job status variable via TCP protocol and Node-RED platform

(ii) IoT data abstraction: cycle time mapping; acquisition of IoT data every second; distribution to BPMS every second

(iii) IoT data sinks: wearable smartwatch interface for human tasks, robot (TCP protocol) where programs are initiated via Service Tasks (HTTP) and Node-RED protocol transformation

The drinks are located in different boxes such that their position varies from drink to drink. We used Node-RED to establish the communication from a Universal Robots UR10 robot (accessible via TCP protocol) via HTTP to Camunda and vice versa. First, a human process participant starts a specific process by means of the smartwatch interface where she chooses a certain drink, e.g., cola, apple juice etc. Having started a new process instance a, Script Task is executed that sends the drink type as a parameter of a HTTP request 
Fig. 6 Wearable process user interface in action

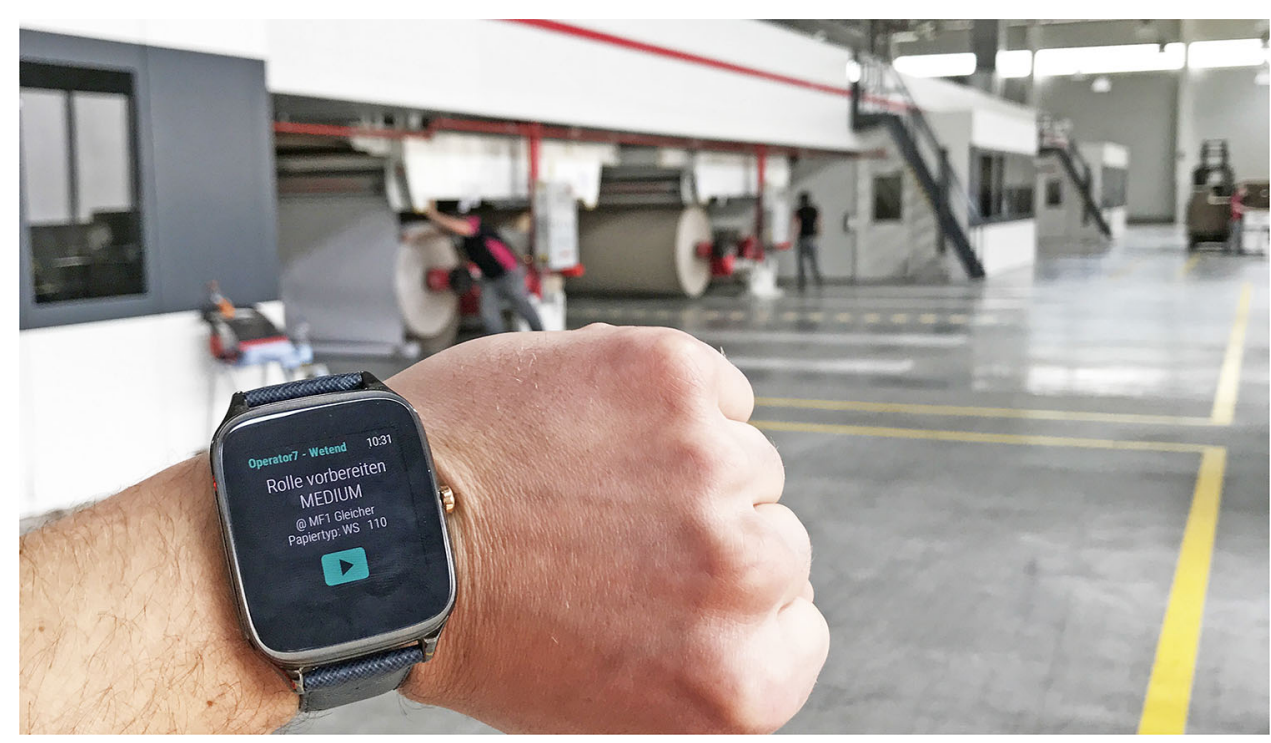

to the Node-RED platform. The parameter is transformed and embedded in a TCP conform message that is sent to the robot. Based on the given parameter, the robot initiates different programs that physically deliver the chosen drink (Fig. 6). Once the robot program has finished, it delivers a TCP message to Node-RED indicating the completion of the job. The IoT data acquisition module finally distributes the status of the job to the BPMS that, in turn, triggers a conditional intermediate event and executes a human task that completes the process. The use case successfully implements the full set of functionality of the integrated bidirectional communication architecture. A screencast illustrating the application use case is available at http://www.maxsyma.de/sosymmaterial/ usecase 2.mov.

\subsection{Specification of SEMI E10 equipment status via smartwatch}

The third use case has been implemented to provide a wearable user interface for the specification of equipment status according to the SEMI E10 standard. For demonstration purposes the process is initiated through a simple proximity sensor. The third application case can be characterized as follows:

(i) Acquired IoT data: proximity sensor signal via TCP protocol

(ii) IoT data abstraction: cycle time mapping; acquisition of IoT data every second; distribution to BPMS every second

(iii) IoT data sinks: wearable smartwatch interface for human tasks, interface to service infrastructure via
Service Tasks (HTTP) and Node-RED protocol transformation

This application was motivated by requests of a wellknown German automation and production company that needed a wearable interface where operators can directly provide information about equipment status in case of machine errors. The actual implemented processes vary from machine to machine; however, the common principle is outlined in Fig. 7, where the signal of a simple proximity sensor is lost as a means to simulate a triggering event like machine errors. In case such an event occurs, two human tasks are triggered and sent to human operators that need to solve and report the problem. Having completed both human tasks, the responsible operator additionally needs to specify the type of error or machine status according to the SEMI E10 standard, respectively. The whole SEMI E10 status tree has been defined as a complex BPMN sub process that is sketched in Fig. 8a. Here, the types and levels of status specifications are modeled as different paths through the process model. In case, the user chooses the status Downtime at the first level, and all other available options at this level are aborted through Boundary Events. At the next level, the operator can choose, a.o., between Unscheduled Downtime and Scheduled Downtime. This selection procedure is continued until the final, i.e., most fine grained selection has been done. The multiple selection options are visualized as a list on the smartwatch interface as shown in Fig. 8 b). The whole SEMI E10 equipment status selection process model can be downloaded at http:// www.maxsyma.de/sosymmaterial/semi10.bpmn. A screencast illustrating the application use case is available at http:// www.maxsyma.de/sosymmaterial/usecase 3.mov. 


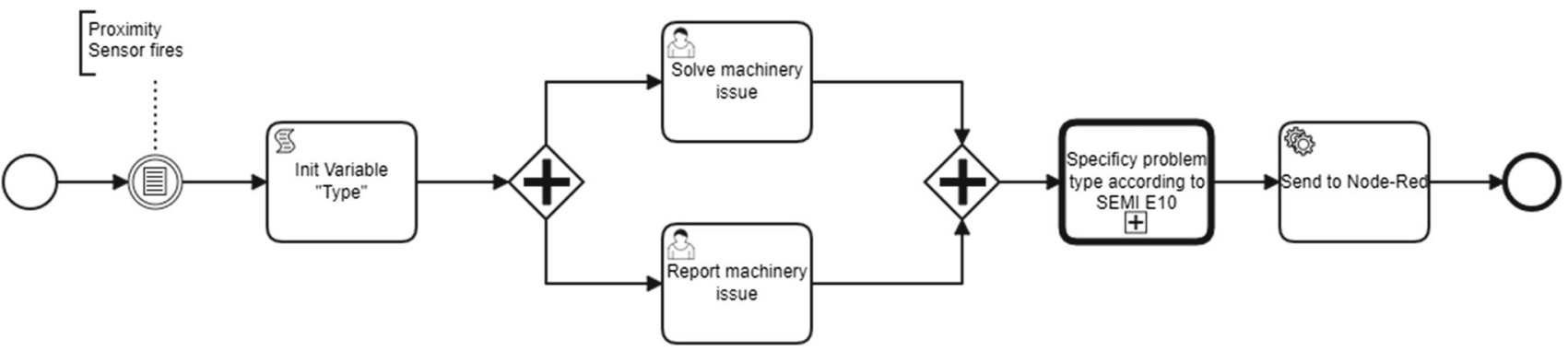

Fig. 7 Specification of machinery issue via smartwatch

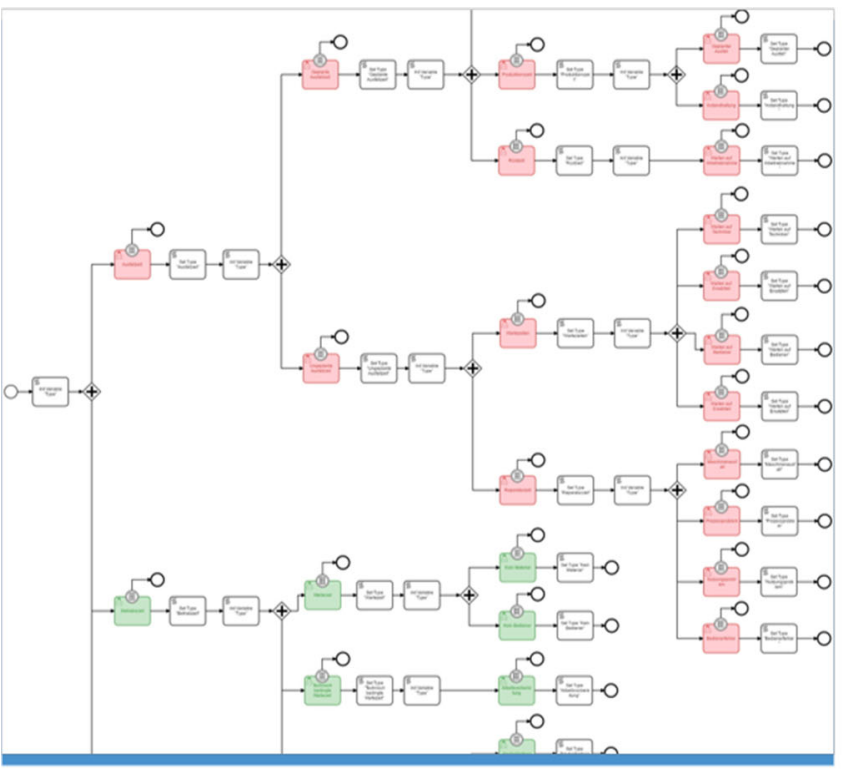

(a)

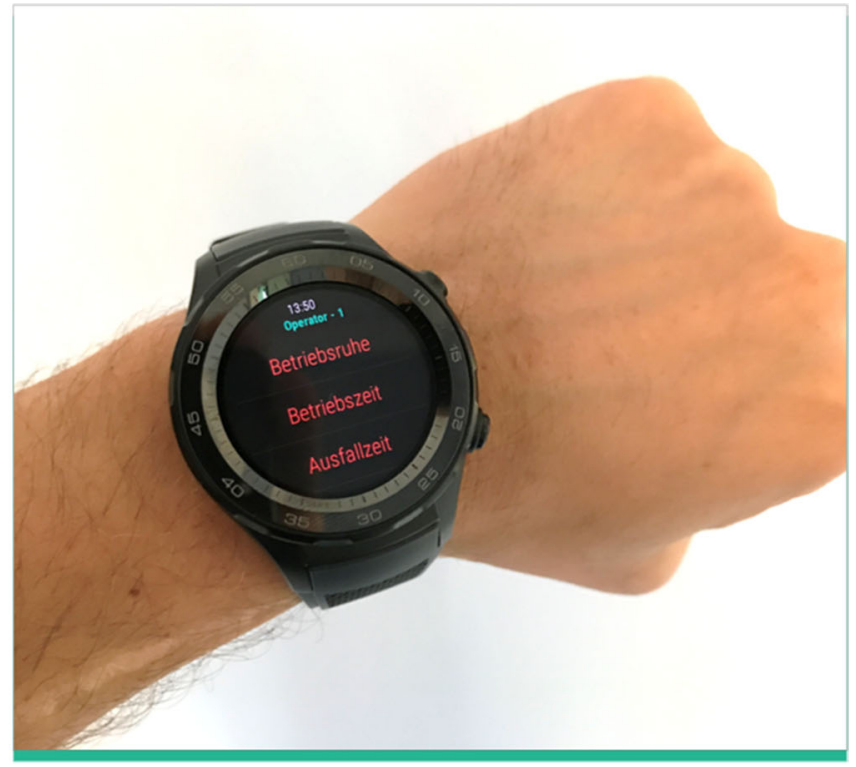

(b)

Fig. 8 Specification of SEMI E10 machinery status: (a) model in BPMN and (b) user interface

\section{Conclusions, implications and future work}

In this paper, we introduced an integrated approach for IoTaware business process execution that tackled several open challenges in this area of applied research. As a fundamental basis for the integration, we introduced an IoT data provenance framework. This technique allows us to consider current IoT data for interaction in arbitrary pre-defined process models that can be enacted by contemporary BPM execution systems. As demanded in many business cases, users need to be notified in real time when new tasks occur. This has been implemented by means of wearable user interfaces with configurable context-specific IoT data provision.

The presented approach combines the recording and analysis of IoT and sensor data with technology of process management. We clearly pointed out that the presented integration can be implemented based on existing concepts and technical solutions. The standard process modeling language BPMN offers adequate modeling elements for the definition and reference of IoT objects and the corresponding real-time data. Existing BPMS like Camunda implements a suitable connector to an IoT protocol like HTTP enabling a bidirectional communication architecture between IoT and process management.

Furthermore, we implemented and evaluated our approach within innovative BPM related use cases, e.g., carried out in production industry. Within this project, we implemented a sensor-enabled wearable process management combining collected sensor data, wearable interfaces and executed BPMN models. First evaluations show that the solution improves the certainty of how and when specific work steps should be carried out and reduces the delay between work steps through mobile and sensor-enabled real-time task provision. Of course the presented solution can be generalized to other industry types as well. As a summary, we want to outline the most important aspects that we learned throughout this extensive project and which factors were mainly contributing to the successful completion of the case. 
- We recognized the advantages that combined IoT and BPM architecture yields compared to traditional information systems for production shop floor. User-specific task coordination based on sensor data as a process oriented solution can provide it seems to be the missing link between production information and operator guidance.

- Modeling IoT-aware business process models is a cumbersome task that requires both deep technical background w.r.t. the IoT-/production system and w.r.t. the used modeling language, e.g., BPMN. To establish a working and accepted solution, an expert in both areas has to tackle this job.

- Understanding BPMN modeled processes by production employees is not as simple as expected. Models have been misinterpreted frequently, and several explanations have been necessary to consolidate a common understanding of the notation and the defined processes.

- As a result, other than planned, BPMN models turned out to be less important as a communication basis for all participating people. Instead, executed IoT-aware processes and concrete task assignments fostered certainty of operators, without knowing the overall flow of work.

There are still several aspects that can be improved and need to be tackled in future research. As outlined in [16], the declarative process modeling approach can serve as a promising alternative paradigm for specifying event-driven IoT-based processes. Therefore, we will investigate and evaluate how IoT-aware processes can be defined, executed and analyzed by existing multi-perspective declarative process technology [46] or how other (declarative) standards like Case Management Model and Notation (CMMN) [47,48] can be adjusted to be used in IoT scenarios. In addition, it is necessary to implement different concepts for a more efficient and secure communication between objects. It would be necessary to establish a more secure communication protocol, e.g., by means of Secure MQTT [49]. Furthermore, the abstraction and pre-processing of raw data stemming from IoT devices needs to be tackled, such that several possibilities for data abstraction are available.

Acknowledgements Open Access funding provided by Projekt DEAL.

Open Access This article is licensed under a Creative Commons Attribution 4.0 International License, which permits use, sharing, adaptation, distribution and reproduction in any medium or format, as long as you give appropriate credit to the original author(s) and the source, provide a link to the Creative Commons licence, and indicate if changes were made. The images or other third party material in this article are included in the article's Creative Commons licence, unless indicated otherwise in a credit line to the material. If material is not included in the article's Creative Commons licence and your intended use is not permitted by statutory regulation or exceeds the permitted use, you will need to obtain permission directly from the copyright holder. To view a copy of this licence, visit http://creativecomm ons.org/licenses/by/4.0/.

\section{References}

1. Dumas, M., La Rosa, M., Mendling, J., Reijers, H.A., et al.: Fundamentals of Business Process Management, vol. 1. Springer, Berlin (2013)

2. Meroni, G., et al.: Artifact-driven process monitoring: dynamically binding real-world objects to running processes. In: CAiSE Forum (2017)

3. Gubbi, J., Buyya, R., Marusic, S., Palaniswami, M.: Internet of things (iot): a vision, architectural elements, and future directions. Future Gener. Comput. Syst. 29(7), 1645-1660 (2013)

4. Mosterman, P.J., Zander, J.: Industry 4.0 as a cyber-physical system study. Softw. Syst. Model. 15(1), 17-29 (2016)

5. Mosterman, P.J., Zander, J.: Cyber-physical systems challenges: a needs analysis for collaborating embedded software systems. Softw. Syst. Model. 15(1), 5-16 (2016)

6. $\mathrm{Da} \mathrm{Xu}, \mathrm{L} ., \mathrm{He}, \mathrm{W} ., \mathrm{Li}, \mathrm{S}$. .: Internet of things in industries: a survey. IEEE Trans. Ind. Inform. 10(4), 2233-2243 (2014)

7. Chen, H., Chiang, R.H., Storey, V.C.: Business intelligence and analytics: from big data to big impact. MIS Q. 36(4), 1165 (2012)

8. Gil, D., Ferrández, A., Mora-Mora, H., Peral, J.: Internet of things: a review of surveys based on context aware intelligent services. Sensors 16(7), 1069 (2016)

9. Chen, M., Mao, S., Liu, Y.: Big data: a survey. Mobile Netw. Appl. 19(2), 171-209 (2014)

10. Lu, Y.: Industry 4.0: a survey on technologies, applications and open research issues. J. Ind. Inf. Integr. 6, 1-10 (2017)

11. Küfner T., Reger, A., Schönig, S.: A PLC-based measuring system for machine crosslinking and monitoring. In: DEStech Transactions on Engineering and Technology Research (2017)

12. Meroni, G., Di Ciccio, C., Mendling, J.: An artifact-driven approach to monitor business processes through real-world objects. In: ICSOC (2017)

13. Meroni, G., et al.: Multi-party business process compliance monitoring through iot-enabled artifacts. Inf. Syst. 73, 61-78 (2018)

14. Schönig, S., Aires, A. P., Ermer, A., Jablonski, S.: Workflow support in wearable production information systems. In: Information Systems in the Big Data Era - CAiSE Forum 2018, Tallinn, Estonia, June 11-15, 2018, Proceedings, pp. 235-243 (2018)

15. Schönig, S., Jablonski, S., Ermer, A.: Iot-basiertes prozessmanagement-mobile benutzerführung in der digitalen fabrik. Informatik Spektrum 42(2), 130-137 (2019)

16. Janiesch, C., Koschmider, A., et al.: The internet-of-things meets business process management: mutual benefits and challenges. arXiv preprint arXiv: 1709.03628 (2017)

17. Soffer, P., Hinze, A., Koschmider, A., Ziekow, H., Ciccio, C.D., Koldehofe, B., Kopp, O., Jacobsen, H., Sürmeli, J., Song, W.: From event streams to process models and back: challenges and opportunities. Inf. Syst. 81, 181-200 (2019)

18. Schönig, S., Ackermann, L., Jablonski, S.: Internet of things meets BPM: a conceptual integration framework. In: Proceedings of 8th International Conference on Simulation and Modeling Methodologies, Technologies and Applications, SIMULTECH 2018, Porto, Portugal, July 29-31, 2018., pp. 307-314 (2018)

19. Schönig, S., Ackermann, L., Jablonski, S., Ermer, A.: An integrated architecture for iot-aware business process execution. In: Enterprise, Business-Process and Information Systems Modeling, pp. 19-34 (2018) 
20. Heinzemann, C., Becker, S., Volk, A.: Transactional execution of hierarchical reconfigurations in cyber-physical systems. Softw. Syst. Model. 18(1), 157-189 (2019)

21. Schönig, S., Jablonski, S., Ermer, A., Aires, A.P.: Digital connected production: wearable manufacturing information systems. In: OTM Workshops (2017)

22. March, S.T., Smith, G.F.: Design and natural science research on information technology. Decis. Support Syst. 15(4), 251-266 (1995)

23. Hevner, A., March, S.T., Park, J., Ram, S.: Design science research in information systems. MIS Q. 28(1), 75-105 (2004)

24. Peffers, K., Tuunanen, T., Rothenberger, M.A., Chatterjee, S.: A design science research methodology for information systems research. J. Manag. Inform. Syst. 24(3), 45-77 (2007)

25. Petrasch, R., Hentschke, R.: Process modeling for industry 4.0 applications - towards an industry 4.0 process modeling language and method. In: International Joint Conference on Computer Science and Software Engineering (2016)

26. Petrasch, R., Hentschke, R.: Towards an Internet-of-Things-aware process modeling method: an example for a house suveillance system. In: Management and Innovation Technology International Conference (2015)

27. Graja, I. et al.: BPMN4CPS: a BPMN extension for modeling cyber-physical systems. In:WETICE, pp. 152-157. IEEE (2016)

28. Meyer, S. et al.: Internet of Things-aware process modeling: integrating IoT devices as business process resources. In: CAiSE, pp. 84-98 (2013)

29. Meyer, S., Ruppen, A., Hilty, L.: The things of the internet of things in BPMN. In: CAISE, pp. 285-297 (2015)

30. Sperner, K. et al.: Introducing entity-based concepts to business process modeling. In: Workshop on BPMN (2011)

31. Domingos, D., Martins, F., Cândido, C., Martinho, R.: Internet of Things aware WS-BPEL business processes context variables and expected exceptions. J. Univers. Comput. Sci. 20(8), 1109-1129 (2014)

32. George, A.A.: Providing context in WS-BPEL processes. Master's thesis, University of Waterloo (2008)

33. George, A.A. Ward, P.A.: An architecture for providing context in ws-bpel processes. In: Conference of Advanced Studies on Collaborative Research (2008)

34. Wieland, M., Kopp, O., Nicklas, D., Leymann, F.: Towards contextaware workflows. In: CAiSE Workshops and Doctoral Consortium, vol. 2, p. 25 (2007)

35. Mateo, J.A. Valero, V., Diaz, G.: BPEL-RF: a formal framework for BPEL orchestrations integrating distributed resources, arXiv:1203.1760 (2012)

36. Schmidt, B., Schief, M.: Towards agile business processes based on the Internet of Things. In: Advanced Manufacturing and Sustainable Logistics, pp. 257-262 (2010)

37. Schobel, J., Pryss, R., Schickler, M., Reichert, M.: A lightweight process engine for enabling advanced mobile applications. In: OTM, pp. 552-569 (2016)

38. Xu, L.D., Xu, E.L., Li, L.: Industry 4.0: state of the art and future trends. Int. J. Prod. Res. 56(8), 2941-2962 (2018)

39. Bousdekis, A., Papageorgiou, N., Magoutas, B., Apostolou, D., Mentzas, G.: A real-time architecture for proactive decision making in manufacturing enterprises. In: On the Move to Meaningful Internet Systems: OTM 2015 Workshops, pp. 137-146 (2015)

40. Li, Q., Jiang, H., Tang, Q., Chen, Y., Li, J., Zhou, J.: Smart manufacturing standardization: reference model and standards framework. In: On the Move to Meaningful Internet Systems: OTM 2016 Workshops, pp. 16-25 (2016)

41. Muller, A., Marquez, A.C., Iung, B.: On the concept of emaintenance: review and current research. Reliab. Eng. Syst. Saf. 93(8), 1165-1187 (2008)
42. Pistofidis, P., Emmanouilidis, C., Koulamas, C., Karampatzakis, D., Papathanassiou, N.: A layered e-maintenance architecture powered by smart wireless monitoring components. In: Industrial Technology (ICIT), pp. 390-395. IEEE(2012)

43. Arnaiz Irigaray, A., Gilabert, E., Jantunen, E., Adgar, A.: Ubiquitous computing for dynamic condition-based maintenance. J. Qual. Maint. Eng. 15(2), 151-166 (2009)

44. Campos, J., Jantunen, E., Prakash, O.: A web and mobile device architecture for mobile e-maintenance. Int. J. Adv. Manuf. Technol. 45(1), 71-80 (2009)

45. Mahdi, R., Jablonski, S., Schönig, S.: Extrinsic dependencies in business process management systems. In: ICEIS (2018)

46. Ackermann, L., Schönig, S., Petter, S., Schützenmeier, N., Jablonski, S.: Execution of multi-perspective declarative process models. In: OTM 2018 Conferences On the Move to Meaningful Internet Systems, pp. 154-172 (2018)

47. Wiemuth, M., Junger, D., Leitritz, M.A., Neumann, J., Neumuth, T., Burgert, O.: Application fields for the new object management group (OMG) standards case management model and notation $(\mathrm{CMMN})$ and decision management notation $(\mathrm{DMN})$ in the perioperative field. Int. J. Comput. Assist. Radiol. Surg. 12(8), 1439-1449 (2017)

48. Schönig, S., Zeising, M., Jablonski, S.: Supporting collaborative work by learning process models and patterns from cases. In: 9th IEEE International Conference on Collaborative Computing: Networking, Applications and Worksharing, pp. 60-69 (2013)

49. Singh, M., Rajan, M.A., Shivraj, V.L., Balamuralidhar, P.: Secure MQTT for internet of things (IoT). In: International Conference on Communication Systems and Network Technologies, pp. 746-751 (2015)

Publisher's Note Springer Nature remains neutral with regard to jurisdictional claims in published maps and institutional affiliations.

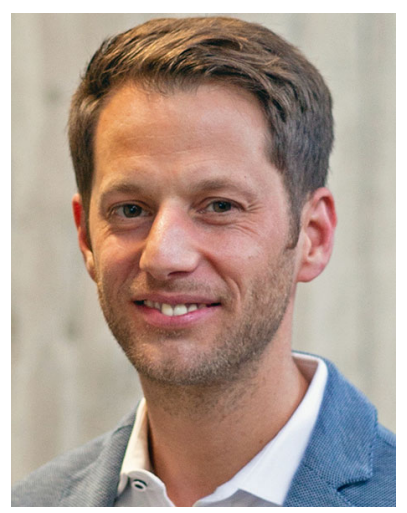

Dr. Stefan Schönig is Professor for Information Systems with the Institute of Management Information Systems at the University of Regensburg in Germany. $\mathrm{He}$ received both the master's degree (with honours) in Applied Computer Science (Engineering/ Computer Science) and the doctoral degree from University of Bayreuth. Before, he held a position as a tenured assistant professor at University of Bayreuth. He was post-doctoral researcher with the Institute for Information Business at WU Vienna (Vienna University of Economics and Business). $\mathrm{He}$ has an established background in BPM/Process Mining and IoT research and has been working in this field for over nine years. $\mathrm{He}$ published extensively in the research area of BPM and Information Systems, both in international conferences and journals. 


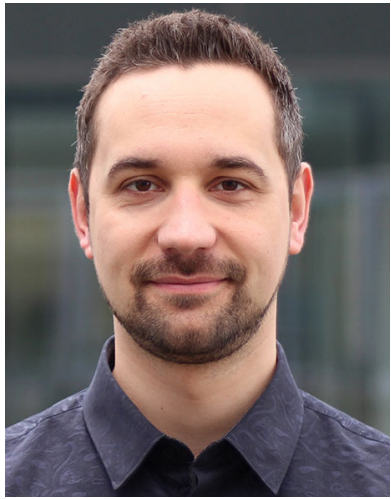

Dr. Lars Ackermann is Assistant Professor of Computer Science with the Institute for Computer Science at University of Bayreuth (Germany). He received the master's degree (with honours) in Computer Science and the doctoral degree from University of Bayreuth. He has an established background in BPM/ Process Mining and has been working in this field for several years. He published extensively in the research area of Business Process Management and Information Systems, both in international conferences and journals.

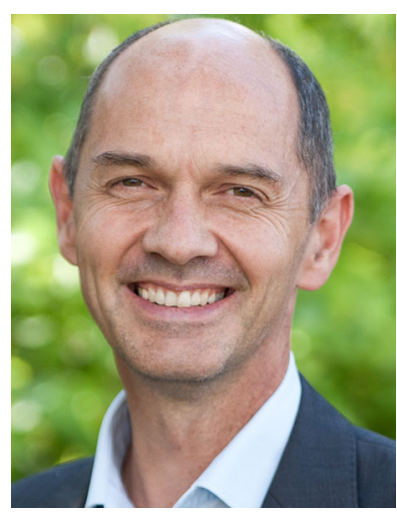

Dr.-Ing. Stefan Jablonski is Full Professor of Computer Science with the Institute for Com-puter Science at University of Bayreuth (Germany). He is head of the chair for Databases and Information Systems. His major research interests include Business Process Management, flexible process enactment technologies, and metamodelling. He has been participating in numerous national and international BPM research as well as industrial projects.

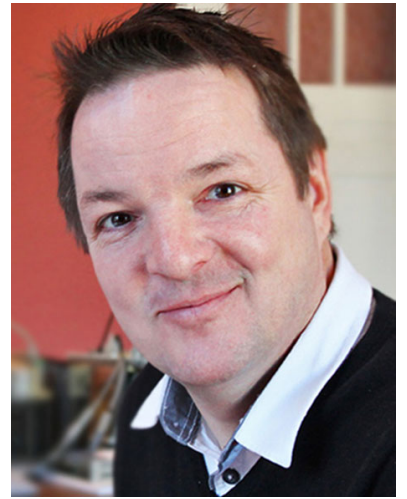

Dipl.-Ing. Andreas Ermer is managing director of the company Maxsyma Automation and Data in Floss in Germany. Before, he was heading the software development department of BHS Corrugated, the world leading company in corrugation equipment industry. He has par-ticipated in several research driven industry projects related to sensor technology, Internet of Things (IoT) equipment as well as BPM integration. Based on his work he has ing international conference proceedings. published various articles in lead- 